\title{
Electrical Arcing at Paddles
}

National Cancer Institute

\section{Source}

National Cancer Institute. Electrical Arcing at Paddles. NCI Thesaurus. Code C63302.

Problem associated with electrical current flowing through a gap between paddles

(conductive surfaces), typically resulting in a visible flash of light. 